\title{
Urinary Incontinence Following Surgery for BPH: the Role of Aging on the Incidence of Bladder Dysfunction
}

\author{
Homero Bruschini, Rogério Simonetti, Alberto A. Antunes, Miguel Srougi \\ Division of Urology (HB, AAA, MS), University of Sao Paulo Medical School, Sao Paulo, Brazil \\ and Division of Urology (RS), Federal University of Sao Paulo, Brazil
}

\begin{abstract}
Purpose: The reported incidence of urinary incontinence (UI) due to bladder dysfunction following surgery of BPH is variable. We described the causes of incontinence in a large group of men that developed this unsual complication and analyzed the influence of age on the prevalence of bladder dysfunction.

Materials and Methods: We wvaluated a total of 125 patients with urinary incontinence following surgical treatment for BPH : Transurethral resection of the prostate ( $81 \mathrm{men})$ and open prostatectomy (44 men). A third group of 21 patients with incontinence following radical prostatectomy was used for comparison. All patients underwent urodynamic analysis. Urethral Sphincter Insufficiency (USI)was defined as involuntary loss of urine induced by Valsalva maneuver in the absence of a detrusor contraction. Bladder dysfunction was defined as detrusor overactivity and/or decreased compliance. Results: Urethral sphincter insufficiency was the most common etiology of urinary incontinence in the three groups of patients. However, bladder dysfunction was observed in $59.3 \%, 56.8 \%$ and $57.1 \%$ of patients who underwent transurethral resection, open prostatectomy and radical prostatectomy, respectively. Median patient age was 69 and 75 years for patients with and without bladder dysfunction, respectively. A logistic regression model for the presence of bladder dysfunction showed that age was a statistically significant predictor.

Conclusions: Urethral Sphincter insufficiency is the main cause of incontinence following surgery for BPH. Bladder dysfunction may be the isolated cause of incontinence in approximately $25 \%$ of patients. The chances of bladder dysfunction rises $5.3 \%$ for each year added to patient age. Patients older than 70 years have twice the probablility of post procedural incontinence.
\end{abstract}

Key words: Prostate; benign prostatic hyperplasia; urinary incontinence; surgery

Int Braz J Urol. 2011; 37: 380-387

\section{INTRODUCTION}

$\mathrm{BPH}$ is the most common benign neoplasm in men (1) and contemporary data shows that about $10 \%$ of these patients will need some form of surgical treatment (2). UI following surgery for BPH is a rare complication. While early incontinence may occur in up to $30-40 \%$ of patients, late incontinence occurs in fewer than $1 \%$ of cases $(3,4)$.

The etiology of post-prostatectomy incontinence can be multifactorial, including USI and/ or Bladder dysfunction (BD) defined as Decreased compliance (DC), or Detrusor Overactivity (DO) (5). USI occurs as a result of injury to the sphincter mechanism and incontinence is usually associated with increase in. $\mathrm{BD}$, which includes detrusor overactivity and/or DC. Moreover, DC is associated with increase in detrusor pressure with storage of urine, which can result in urinary incontinence.

However, while UI following RP, is mainly attributed to SI, $(5,6)$ the prevalence of BD among patients with UI following surgery for BPH may 
be significantly higher (7). Some studies have reported that more than $60 \%$ of these cases are attributed to $\mathrm{BD}$, probably as a result of the long-lasting obstructive process (8).

Due to the inconsistent reports linking BD to post-prostatectomy incontinence, (7-9) and the impact of BD on the outcome of treatment, we reported the causes of UI in a relatively large group of men with this rare complication following surgery for BPH and compared them to a group of patients with UI following RP. We also analyzed the influence of age on the prevalence of BD.

\section{MATERIALS AND METHODS}

A total of 125 patients referred from various centers with UI following surgical treatment for BPH (TURP in 81 and OP in 44) were evaluated at our Urodynamics laboratory during the last 19 years. A third group of 21 patients with UI following RP for treatment of prostate cancer was used for comparison. Mean elapsed time between prostatectomy and urodynamics evaluation was 21 months (range 6 to 168 months).

All patients provided a detailed clinical history which included age, time interval since the prostatectomy, presence of urgency or stress incontinence and concurrent medications. Severity of incontinence varied from use of one pad per day to constant leakage and all men were disappointed with the complication. A focused neurological examination was performed at presentation and no patient presented a potential neurological cause for their urinary dysfunction.

All patients underwent urodynamic analysis as previously described (10). Uroflowmetry was performed when possible. Peak flow rate, voiding time and volume were recorded. The patients were then catheterized and post-void residual volume was measured. Cystometrogram comprised multichannel water cystometry with simultaneous measurement of vesical and abdominal pressure. Intravesical, intra-abdominal and detrusor pressures, filling and volume voided, were measured.. International Continence Society recommended definitions were applied (11). DO was defined as any involuntary bladder contraction occurred during filling with a pressure rise of $15 \mathrm{cmH} 2 \mathrm{O}$ or more, or involuntary contraction of any magnitude associated with urgency. Bladder compliance was calculated by dividing the bladder capacity in $\mathrm{cc}$ by detrusor pressure in $\mathrm{cmH} 2 \mathrm{O}$ at cystometric capacity. A value of less than $12.5 \mathrm{cc} / \mathrm{cmH} 2 \mathrm{O}$ was considered DC (12). USI was defined as involuntary loss of urine induced by Valsalva maneuver in the absence of a detrusor contraction. BD was defined as DO and/or DC.

Urinary incontinence was defined as an involuntary loss of urine passively or stress induced during the urodynamic evaluation. With the patient in the supine position, the bladder was filled to 200-250 mL (Filling rate of $50 \mathrm{~mL}$ per minute) and various provocative maneuvers were performed. If no leakage occurred, stress test was repeated at $50 \mathrm{~mL}$ increments until maximum capacity. During the filling any overactivity or DC was noted. If no leakage was detected, the urethral catheter was removed and the patient was asked to perform Valsalva maneuver in the standing position. All urodynamic studies were performed by the same physician. Review of each study was done by independent third party, to eliminate any artifacts and to determine accurately the urodynamic diagnosis.

For statistical analysis patients were divided into groups based on type of surgery and urodynamic diagnosis. Analysis of patients characteristics according to the type of surgery were performed through the ANOVA, Kruskal Wallis and Fisher's exact tests. Relationships between patient age and urodynamic variables were calculated through the Pearson's correlation coefficient. For analysis of patient age according to the presence of BD we also used the Student's T test. Finally, the influence of age on urodynamic findings was analyzed using a logistic regression model. Statistical significance was defined as a $\mathrm{p}<0.05$.

\section{RESULTS}

Patients age ranged from 45 to 88 years old (mean 69 y.o.). Analysis of patient's characteristics according to the type of surgery showed that patients with UI who underwent OP tended to be 
older and had greater rates of compliants related to stress UI. Median urinary flow rates were greater among patient who underwent RP, but these results were only marginally significant. Median urethral functional length was shorter among patients who underwent RP (Table-1). Other variables were not statistically different between the three groups. tion with USI in 20 patients (13.7\%). DC was the sole cause of incontinence in 13 patients $(8.9 \%)$ and occurred in combination with SUI in 30 patients $(20.5 \%)$. While almost $14 \%$ of patients who underwent TURP presented DC as the sole cause of UI, no patient who underwent RP had this finding; however this difference was only marginally

Table 1 - Characteristics of the patients according to the type of surgery.

\begin{tabular}{|c|c|c|c|c|}
\hline & TURP $(n=81)$ & $\mathrm{OP}(\mathrm{n}=44)$ & $\operatorname{RRP}(n=21)$ & $\mathbf{p}$ \\
\hline Mean age (years) & $69.0( \pm 9.1)$ & $72.0( \pm 6.9)$ & $67.0( \pm 6.3)$ & $0.07^{*}$ \\
\hline \multicolumn{5}{|l|}{$(\min -\max )$} \\
\hline \multicolumn{5}{|l|}{ Complaint } \\
\hline Stress UI & $43.2 \%$ & $72.7 \%$ & $52.4 \%$ & \multirow[t]{3}{*}{$0.004 * *$} \\
\hline Urgency UI & $16.0 \%$ & $2.3 \%$ & $2.3 \%$ & \\
\hline TotalUI & $39.5 \%$ & $25.0 \%$ & $47.6 \%$ & \\
\hline Median urinary flow $(\mathrm{mL} / \mathrm{s})$ & 9.0 & 10.0 & 12.0 & \multirow[t]{2}{*}{$0.087^{* * *}$} \\
\hline$(\min -\max )$ & $(3-24)$ & $(2-35)$ & $(8-16)$ & \\
\hline Median bladder capacity $(\mathrm{mL})$ & 350 & 350 & 350 & \multirow[t]{2}{*}{$0.608 * * *$} \\
\hline$(\min -\max )$ & $(50-900)$ & $(50-600)$ & $(100-500)$ & \\
\hline Urethral leak point pressure & 42 & 37.5 & 40 & \\
\hline$(\mathrm{cm} \mathrm{H} 2 \mathrm{O})$ & $(10-200)$ & $(0-200)$ & $(2-90)$ & \\
\hline Median urethral functional length $(\mathrm{cm})(\mathrm{min}$ & $3(1-6.5)$ & $3(1-6)$ & $2(1-3.5)$ & $0.006 * * *$ \\
\hline$-\max )$ & & & & \\
\hline
\end{tabular}

*ANOVA / **Fisher exact test / ***Kruskal Wallis test

The etiology of post-prostatectomy incontinence according to urodynamic analysis was unrelated to the type of surgery. USI was the most common etiology of urinary incontinence in the three groups, and was observed in $66.7 \%, 79.6 \%$ and $85.7 \%$ of patients who underwent TURP, OP and RP respectively $(\mathrm{p}=0.126)$. USI alone was found in 56 patients $(38.4 \%)$ and was present in combination with $\mathrm{BD}$ in additional 51 patients $(34.9 \%)$. USI was considered the main cause of UI in those patients with associated BD because of their clinical complains (stress urinary incontinence without symptoms of urgency). BD consisting of DO and/or DC was detected in a total of 85 patients $(58.2 \%)$. DO was the isolated cause in 21 patients $(14.4 \%)$ and was found in combina- significant. One patient presented poor compliance and DO in combination with SUI. One patient had bladder outlet obstruction and overflow incontinence. In three patients incontinence was not demonstrated on urodynamic evaluation (Table-2).

Analysis of patient age according to the presence of BD showed that median patient age was 72 and 68 years old among patients with and without BD respectively $(\mathrm{p}=0.020)$ (Figure-1). Correlation of patient age and complaint of stress or total UI demonstrated that median patient age was 69 and 75 years old for patients with and without incontinence issues, respectively $(\mathrm{p}=0.031)$. The median patients age was 68 and 73 years old for patients with USI and without USI documented with uodynamic studies, respectively $(\mathrm{p}<0.001)$ 
Table 2 - Cause of UI according to the type of surgery.

\begin{tabular}{lcccc}
\hline Cause & TURP & OP & RP & p \\
\hline DC & $13.6 \%$ & $4.5 \%$ & 0 & 0.086 \\
DO & $16.0 \%$ & $13.6 \%$ & $9.5 \%$ & 0.848 \\
DO + SI & $9.9 \%$ & $20.5 \%$ & $14.3 \%$ & 0.242 \\
SI & $37.0 \%$ & $40.9 \%$ & $38.1 \%$ & 0.943 \\
SI + DC & $18.5 \%$ & $18.2 \%$ & $33.3 \%$ & 0.304 \\
SI + DO + DC & $1.2 \%$ & 0 & 0 & $>0.999$ \\
Normal & $2.5 \%$ & 0 & $4.8 \%$ & 0.253 \\
Obstruction & $1.2 \%$ & $2.3 \%$ & 0 & $>0.999$ \\
\hline
\end{tabular}

(Figure-2). Associations of age with urodynamic parameters through the Pearson correlation test only showed that age was inversely associated with urinary flow. The other parameters presented lower correlation coefficients (Table-3).

A logistic regression model for the presence of BD considering patients age and type of surgery revealed that age was a statistically significant predictor of outcome. (Table-4). These results demonstrate that each additional year of age increases BD by about $5.3 \%$. Likewise, when patients with a 10 year difference in age are compared, the chances of BD increases by $67 \%$. BD was 2.3 times more likely to occur in older patients $>70$ years old $(\mathrm{p}=$ 0.017 ; OR $=2.326$ ). The type of the surgery had no significance in these results. In fact, the proportion of patients with BD was $59.3 \%, 56.8 \%$ and $57.1 \%$ among patients who underwent TURP, OP and RP respectively $(\mathrm{p}=0.960)$.

\section{DISCUSSION}

Post-prostatectomy urinary incontinence, although often temporary, is a devastating complication with a major impact on quality of life. In the present study we demonstrated that despite patients who underwent OP presented greater rates of incontinence related to stress UI, the etiology of UI according to urodynamic findings were statistically similar between the three groups of patients. Patients who underwent TURP presented higher rates of UI due to DC when compared to patients who underwent OP or RP, but this figure was only marginally significant.

Post-prostatectomy incontinence may be a temporary impairment which may spontaneously resolve. Most patients recover urinary continence within the first 6 months following surgery. Early incontinence is usually related to urge incontinence, either because of irritative symptoms due to the prostatic fossa healing and associated urinary tract infections or detrusor overactivity caused by long-lasting BPH (3). In the present series, urodynamic studies were performed after the 6th postoperative month.

Incontinence that persists longer than six months may require complete investigation including an urodynamic evaluation. Many factors have been implicated in the etiology of post-prostatectomy incontinence. They include BD, such as DC and/or DO and damaged sphincter mechanism. In the present study more than $70 \%$ of cases of UI were attributed to USI. These cases are usually caused by iatrogenic trauma of the external sphincter. Particularly, in cases that undergo TURP, which were the majority in our series, the lesion usually occurs ventrally (at 12 o'clock), where the Verumontanum is not visible. Also, there is an increased risk of sphincter injury if the Verumontanum has already been resected (3).

Conflicting reports in the literature make it difficult to confirm the importance of BD on the pathogenesis of UI. In our series, BD was detected in $58.2 \%$ of the patients and was the isolated cause 
Figure 1 - Mean patients age according to the prevalence of bladder dysfunction $(p=0.020)$.

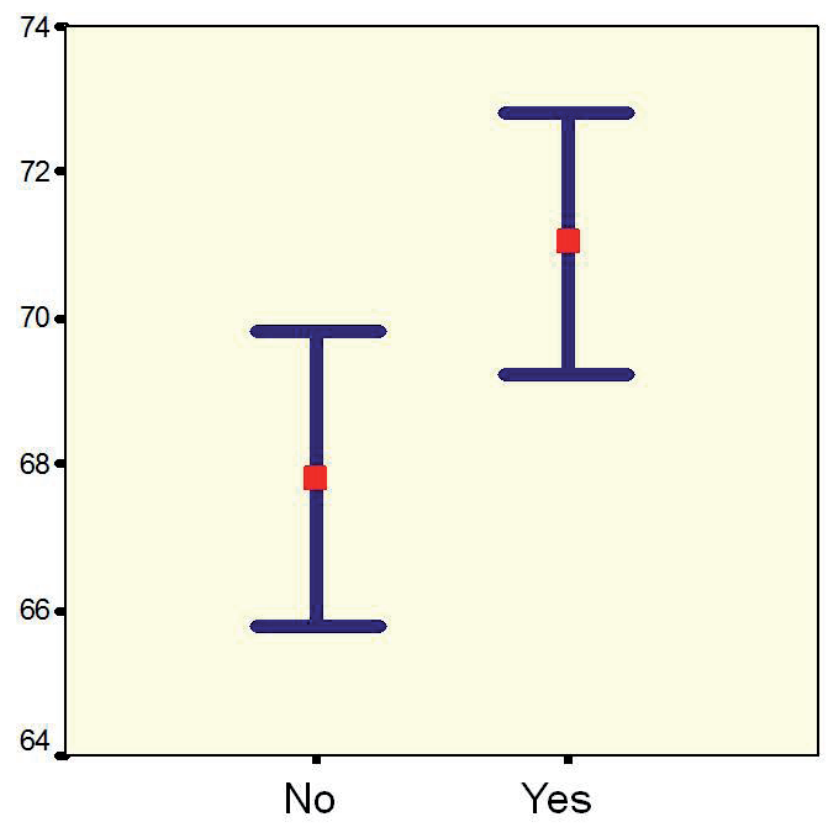

\section{Bladder dysfunction}

of UI in approximately $25 \%$ of patients. Although only $9.5 \%$ of patients who underwent RP had UI due to BD, these figures were only marginally significant (Table-2). Similarly to our results, a study of 60 men with a mean age of 64.8 years who underwent RP and acquired post-prostatectomy UI, USI was demonstrated in $90 \%$ of cases. A component of BD was seen $45 \%$ of cases (mainly due to $\mathrm{DO})$, but incontinence was actually a result of $\mathrm{BD}$ in only $27 \%$ of patients (5).

Other series have analyzed patients who underwent surgery for BPH regarding post-operative continence rates. An analysis of 56 incontinent patients, 31 who had undergone TURP and 25 who had undergone RP, showed that patients in the TURP group were older and had higher incidence of DO. Interestingly, the most common etiology for UI, was DO alone. DO was present in $61 \%$ of cases, including $77 \%$ after TURP and $40 \%$ after RP. Stress incontinence alone was present in only $5 \%$ of patients, including 3\% after TURP and $8 \%$ after $\mathrm{RP}$, and DO with stress incontinence was present in
$34 \%$ of cases, including $19 \%$ after TURP and 52\% after RP (8). Another retrospective review of the records of 56 patients with post-prostatectomy incontinence after surgery for BPH showed that $43 \%$ were found to have complex incontinence (either mixed or associated with bladder outlet stenosis), requiring combined treatment. Forty one percent had a simple type of incontinence, i.e., pure USI or DO. Three patients had residual adenoma and 1 had a urethral stricture, while $9 \%$ remained unclassified (9). Higher rates of USI were found by Winters et al., (7) who analyzed 92 patients with UI (65 after RP and 27 after TURP) with multichannel videourodynamic testing performed at least 1 year after prostatectomy. USI was detected in $92 \%$ of cases and despite DO was a common finding, occurring in $37 \%$ of patients, it was found to be the sole cause of UI in only $3.3 \%$ of cases. There was no statistically significance in the incidence of USI after RP or TURP; however, TURP patients had a significantly higher incidence of DO.

In the present study we also analyzed the influence of patient age on the etiology of UI. The influence of age on recovery of urinary continence

Figure 2 - Mean patients age according to the presence of urinary sphincter insufficiency $(p<0.001)$.

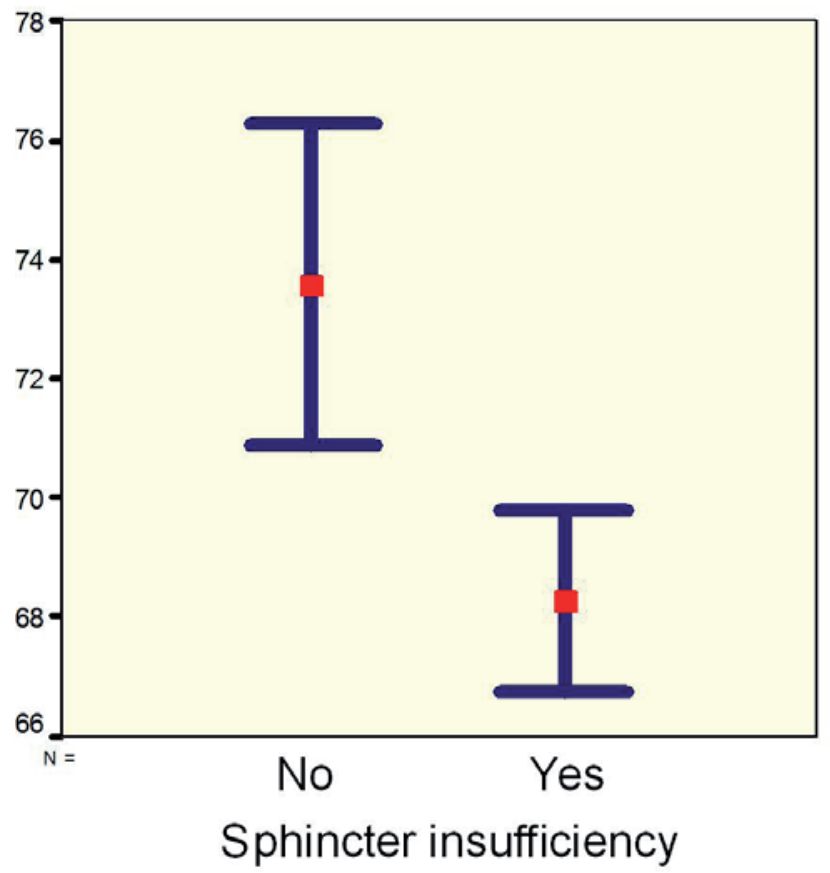


Table 3 - Correlation coefficients of patient age with urodynamic parameters.

\begin{tabular}{lcc}
\hline Variable & Pearson's correlation & p \\
\hline Flow rate & -0.184 & 0.037 \\
Urethral leak point pressure & 0.128 & 0.127 \\
Functional lengh & 0.045 & 0.603 \\
Post-void residual volume & 0.132 & 0.112 \\
Bladder capacity & -0.124 & 0.137 \\
\hline
\end{tabular}

Table 4 - Logistic regression model for the presence of UI.

\begin{tabular}{cccc}
\hline Variable & $\mathbf{p}$ & Odds ratio & CI (95\%) \\
\hline Age & 0.018 & 1.053 & {$[1.009 ; 1.099]$} \\
OP / TURP & 0.534 & 0.784 & {$[0.364 ; 1.689]$} \\
RP / TURP & 0.779 & 1.013 & {$[0.377 ; 2.724]$} \\
\hline
\end{tabular}

has been studied mainly among patients who underwent RP, and conflicting results have been shown in the literature. Eastham et al. (13) studied risk factors for UI following RP in 581 patients who were previously continent. They concluded in a multivariate analysis that the factors that were independently associated with increased chance of regaining continence were decreasing age, a modification in the technique of anastomosis (introduced in 1990), preservation of both neurovascular bundles and absence of an anastomotic stricture. Conversely, in the study of Catalona and Basler (14) who analyzed 784 patients that underwent RP, continence was regained in $94 \%$ of cases and did not correlate with patient age.

The present study analyzed the influence of age on the etiology of UI among patients who underwent surgery for BPH showed that despite age had low correlation coefficient rates with urodynamic parameters, patients who presented BD were 3.2 years older than the ones who did not present this abnormality. Furthermore, the chances of presenting BD rises 5.3\% for each year of age and patients older than 70 years old had twice the chance of presenting $\mathrm{BD}$, no matter which type of surgery they had. These figures could explain why older patients may sometimes present greater rates of UI, or longer periods for recovery of UI. The association of aging and $\mathrm{BD}$ in men is well known, and studies have shown similar occurence in elderly women.

Limitations of the present study include the retrospective nature of this report and the relatively small number of patients with UI following RP precluded an accurate comparison of urodynamic parameters. Preoperative urodynamic data were not available, since most patients were referred from other medical centers. Some patients were submitted to urodynamic testing six months after surgery, and as reported by Giannantoni et al. (15), improvement in compliance and contractility may occur after this period in patients submitted to radical prostatectomy. However, to our knowledge, this is the largest study to analyze patients with late UI following surgery for $\mathrm{BPH}$.

\section{CONCLUSIONS}

Urinary Stress Incontinence is the main cause of post-prostatectomy incontinence following surgery for BPH. However, BD may be the isolated cause of UI in approximately $25 \%$ of patients and coexist with USI in $30 \%$ of cases. Older patients have greater rates of BD and patients older than 70 
years of age may be considered a high risk group. Urodynamic evaluation is essential to identify the etiology of post-prostatectomy incontinence and to optimize the outcome of treatment.

\section{REFERENCES}

1. AUA Practice Guidelines Committee: AUA guideline on management of benign prostatic hyperplasia (2003). Chapter 1: Diagnosis and treatment recommendations. J Urol. 2003; 170: 530-47.

2. McConnell JD, Bruskewitz R, Walsh P, Andriole G, Lieber M, Holtgrewe HL, et al.: The effect of finasteride on the risk of acute urinary retention and the need for surgical treatment among men with benign prostatic hyperplasia. Finasteride LongTerm Efficacy and Safety Study Group. N Engl J Med. 1998; 338: 557-63.

3. Rassweiler J, Teber D, Kuntz R, Hofmann R: Complications of transurethral resection of the prostate (TURP)--incidence, management, and prevention. Eur Urol. 2006; 50: 969-79; discussion 980.

4. Wendt-Nordahl G, Bucher B, Häcker A, Knoll T, Alken P, Michel MS: Improvement in mortality and morbidity in transurethral resection of the prostate over 17 years in a single center. J Endourol. 2007; 21: 1081-7.

5. Ficazzola MA, Nitti VW: The etiology of post-radical prostatectomy incontinence and correlation of symptoms with urodynamic findings. J Urol. 1998; 160: 1317-20.

6. Wei JT, Dunn RL, Marcovich R, Montie JE, Sanda MG: Prospective assessment of patient reported urinary continence after radical prostatectomy. J Urol. 2000; 164: 744-8.

7. Winters JC, Appell RA, Rackley RR: Urodynamic findings in postprostatectomy incontinence. Neurourol Urodyn. 1998; 17: 493-8.

8. Goluboff ET, Chang DT, Olsson CA, Kaplan SA: Urodynamics and the etiology of post-prostatectomy urinary incontinence: the initial Columbia experience. J Urol. 1995; 153: 1034-7.

9. Theodorou C, Moutzouris G, Floratos D, Plastiras D, Katsifotis C, Mertziotis N: Incontinence after surgery for benign prostatic hypertrophy: the case for complex approach and treatment. Eur Urol. 1998; 33: 370-5.

10. Almeida FG, Bruschini H, Srougi M: Correlation between urethral sphincter activity and Valsalva leak point pressure at different bladder distentions: revisiting the urethral pressure profile. J Urol. 2005; 174: 1312-5; discussion 1315-6.

11. Abrams P, Blaivas JG, Stanton SL, Andersen JT: The standardisation of terminology of lower urinary tract function. The International Continence Society Committee on Standardisation of Terminology. Scand J Urol Nephrol Suppl. 1988; 114: 5-19.

12. Toppercer A, Tetreault JP: Compliance of the bladder: an attempt to establish normal values. Urology. 1979; 14: 204-5.

13. Eastham JA, Kattan MW, Rogers E, Goad JR, Ohori $\mathrm{M}$, Boone TB, et al.: Risk factors for urinary incontinence after radical prostatectomy. J Urol. 1996; 156: 1707-13.

14. Catalona WJ, Basler JW: Return of erections and urinary continence following nerve sparing radical retropubic prostatectomy. J Urol. 1993; 150: 9057.

15. Giannantoni A, Mearini E, Di Stasi SM, Mearini L, Bini V, Pizzirusso G, et al.: Assessment of bladder and urethral sphincter function before and after radical retropubic prostatectomy. J Urol. 2004; 171: 1563-6.

Accepted after revision:

July 30, 2010

\section{Correspondence address:}

Dr. Alberto Azoubel Antunes

Rua Barata Ribeiro, 490 / 76

São Paulo, SP, 01308-000, Brazil

E-mail: antunesuro@uol.com.br

\section{EDITORIAL COMMENT}

The purpose of the study is to assess the relative role of bladder dysfunction and urinary sphincter deficiency following prostate surgery. This interesting subject is difficult and articles have already been published on it. However many 
factors may be involved in incontinence following surgery for BPH. The role of age is one of them, but age may be a cause of sphincter deficiency as well as bladder dysfunction or both. The analysis is complex and conclusions difficult to draw. The limit of this study was to be a retrospective analysis, and to have been carried out only after surgery without comparative data before surgery. Concerning the follow-up period, a minimal 6 months interval between surgery and urodynamics was probably not enough in some patients, usually a 12 months was reported. However the authors presented an interesting study and have to be encouraged to initiate a prospective study.

\author{
Dr. Philippe Grise \\ Department of Urology \\ Rouen University Hospital-Charles Nicolle \\ rue de Germont \\ 76031, Rouen, France \\ Fax: + 332 3288-8205 \\ E-mail:philippe.grise@chu-rouen.fr
}

